\title{
Comparing the TPB and the T-D-I-B framework
}

\author{
Warren B. Miller*
}

\section{Introduction}

There is little doubt that the Theory of Planned Behaviour (TPB) has been one of the most successful of social and behavioural science theories in the prediction of psychological and behavioural phenomena across all behavioural domains. Its domain-general success, however, suggests that the TPB may have limitations when it comes to specific domains such as human reproductive behaviour. An important reason for such limitations is that we as behavioural scientists want more than prediction; because we are relatively youthful as a science, we want constructs and hypotheses that take us beyond prediction to the generation of new understanding. I believe one way that this can best be accomplished is by linking the unique domain-specific phenomena we study to constructs from other domains, especially those that operate at different levels of organisation such as the biological and the social levels.

In my own work I have developed a theoretical framework for understanding human reproductive behaviour that shares many generic features with the TPB. This Traits-Desires-Intentions-Behaviour or T-D-I-B framework is built around the sequence of motivational dispositions and conscious states that lead humans to behave so as to have or avoid having children. The sequence begins with both positive and negative childbearing motivational traits; these traits lead to various desires for or against having children, which in turn contribute to various corresponding fertility intentions. The sequence concludes with behaviours that are oriented either to the achievement or avoidance of pregnancy and childbearing. The TPB has a similar four-step sequence. First, there are beliefs about the outcomes of a specific behaviour, how others feel about that behaviour, and whether certain factors affecting the behaviour are present; second, these beliefs are shaped by evaluation processes to produce attitudes and subjective norms with regard to the behaviour as well as one's perceived degree of control over it; third, there are intentions to perform the behaviour; and finally there is the behaviour itself. I will structure my commentary below so as to compare these two frameworks across each of their corresponding four steps. Although this debate is about the TPB, I am far more conversant with the T-D-I-B framework. I will therefore focus on the T-D-I-B perspective in my discussion with the goal of

\footnotetext{
* Warren B. Miller, Transnational Family Research Institute, 229 Wixon Avenue, Aptos, California, 95003-4626 USA. Email: rennie@tfri.org
} 
identifying ways of thinking about the behavioural domain of human reproduction that may broaden and strengthen the theoretical and conceptual approaches in the next generation of the TPB. I also hope that the dialogue initiated in this publication will benefit the T-D-I-B framework and, ultimately, lead to an expansion and greater integration of all the current kindred theories of fertility motivation and reproductive behaviour. I will begin with a discussion of the behaviours to be predicted and then move backward in the chain of motivational constructs.

\section{Behaviours}

Throughout the animal kingdom reproductive behaviours are first and foremost sexual behaviours. Human reproductive behaviour is unique in that we understand the relationship between the occurrence and timing of sexual intercourse and pregnancy, which enables us to organise our sexual behaviour in order to achieve or prevent conception. This means that reproductive decisions, and thus the intentions that are a fundamental part of them, are taken in the context of a sexual relationship and, quite often, in the course of sexual behaviour during which people are under the influence of the sexual and relationship motivations that drive it. In this context there are basically three types of conception-oriented action that may be pursued: trying to avoid conception; trying to achieve it; and not trying to do anything about it. Each of these three action types has its own behavioural gradient. For those trying to avoid conception, there is a gradient from low to high effectiveness of contraceptive use (Miller and Pasta 1996c). For those trying to achieve conception, there is a gradient from passive proception, in which contraceptive efforts are discontinued in order to achieve conception, to active proception, in which various sexual behaviours that improve the chances of conception are pursued (Miller 1986; Miller and Pasta 1996a). Finally, for those not trying to do anything, there is a group of people who say they do not care one way or the other about getting pregnant (Miller and Pasta 2002; McQuillan et al. 2011). Both evidence from research (Miller 1986; Miller and Pasta 1995) and common sense indicate that this group has separate gradients of intent towards avoiding conception and toward achieving it.

In Figure $1 \mathrm{I}$ have placed eight categories of conception-oriented behaviour based on these three groups along the $\mathrm{x}$ axis, arranging them in a continuum that corresponds closely to the intentionality continuum described by Bachrach and Newcomer (1999). The headings at the top of the figure correspond to the three types of conception-oriented action described above and the symbols along the $\mathrm{x}$ axis at the bottom of the figure indicate the various categories within each type of action. Thus there are three categories of contraceptive behaviour, designated by a ' $C$ ' with progressively higher subscripts - indicating more effective contraceptive use - moving toward the right-hand margin of the figure. The choice of three categories is somewhat arbitrary - there could be two, four, or more; however, they correspond well to the divisions I have used in my own research, 
characterising the effectiveness of contraceptive use as low, medium and high. Moving toward the left-hand margin of the figure are two categories of proceptive behaviour, designated by a ' $\mathrm{P}$ ' with progressively higher subscripts - indicating a more effective pursuit of proception. Again the number of categories is somewhat arbitrary but corresponds to the passive/active distinction I have made in my research. Finally, there are three categories where the behaviour is in itself neither contraceptive nor proceptive. The middle category designated with a zero has neither contraceptive nor proceptive intent, whereas the zeros with the ' $\mathrm{P}$ ' and ' $\mathrm{C}$ ' subscripts represent transitional categories where a person has the intent to use contraceptive or proceptive measures but is not in fact implementing the relevant behaviour at the time.

\section{Figure 1:}

Ranges in the strength of both contraceptive intentions ( $I_{C}$, dashed lines) and proceptive intentions ( $I_{P}$, solid lines), based on hypothetical values assigned to each of eight categories of conception-oriented behaviour

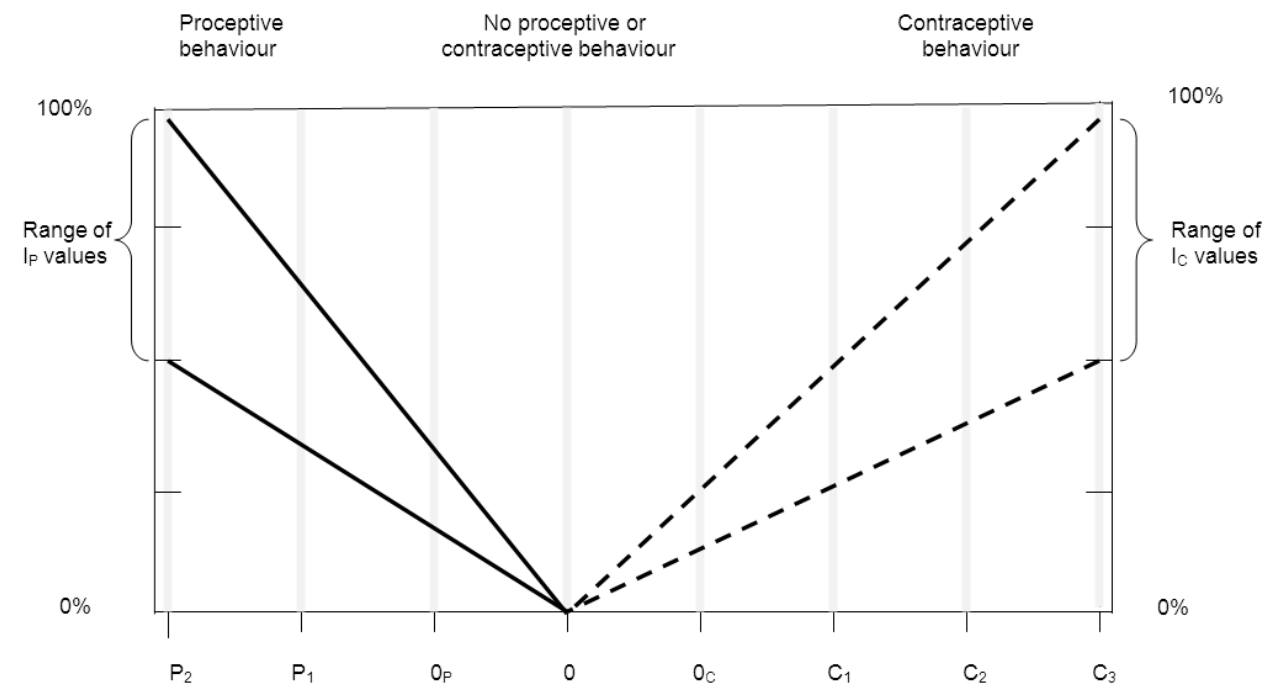

The TPB has been most successful in the measurement and prediction of contraceptive behaviours such as condom use (e.g. Sutton et al. 1999; Albarracin et al. 2001). There has been relatively little in the literature to date on the measurement and prediction of fertility behaviour by researchers using the TPB (Philipov et al. 2009), although several promising studies were reported at the recent Vienna Institute of Demography conference (2010) and Ajzen (2010) also indicated that a study by Klobas and Ajzen was in preparation. The continuum of behaviours indicated along the $\mathrm{x}$ axis of Figure 1 represents a bipolar scale that ranges from a pole of consistent and effective contraceptive behaviour on the 
right to a pole of consistent and effective proceptive behaviour on the left. The research strategy of the available TPB concerned with predicting these behaviours has been to focus on behaviour at one pole of the continuum-chiefly at the contraceptive end. This is not an unreasonable strategy because predicting the $\mathrm{C}_{1}$ through $\mathrm{C}_{3}$ behaviours may be the most important one for prediction purposes in any given population. However, this does not increase our understanding of the full continuum and the shifts in intentions that occur within it as people travel through their reproductive careers and encounter situational changes and constraints.

\section{Intentions}

In Figure $1 \mathrm{I}$ have also shown hypothetical plots for both contraceptive intentions (dashed lines) and proceptive intentions (solid lines). The right and left y axes of the figure represent the strength of intentions with a metric that goes from a high value of $100 \%$ to a low one of $0 \%$. Contraceptive intentions are indicated for the behavioural categories $\mathrm{C}_{3}$ to 0 , with the upper and lower dashed lines indicating an approximate range of values that might reasonably be found in samples of people from each of the five contraceptive behavioural categories. Proceptive intentions are indicated for the behavioural categories $\mathrm{P}_{2}$ to 0 , with the upper and lower solid lines similarly indicating an approximate range of values for the four proceptive behavioural categories. The range of values for both contraceptive and proceptive intentions is large at $\mathrm{C}_{3}$ and $\mathrm{P}_{2}$ and narrows to $0 \%$ at the zero behavioural category. It makes sense that the upper border of both the contraceptive and proceptive ranges approaches the $100 \%$ strength metric because we would expect very strong intentions to result in very effective behaviour. The placement of the low borders takes partner and situational factors into account; for example, some people may have intentions of only modest strength but be very effective in the pursuit of their conception-oriented goal as a result of their partners' behaviours.

It can be seen in Figure 1 that there is no overlap in the ranges of contraceptive and proceptive intentions. This makes sense because it is not possible to both try not to conceive and try to conceive at the same time, although it is possible in some instances for intentions to jump back and forth over a short period of time, especially if the person is involved with more than one partner. The figure also shows a large area in the middle where there are no intentions. The significance of this can be understood if we look at some US data indicating what proportion of people of reproductive age fall into the seven behavioural categories. Finer et al. (2007) reported a frequency analysis of data from the 2002 National Survey of Family Growth of US women's responses to the following question about the pregnancies they experienced during the previous five years $(2,719$ pregnancies in the sample): "If you had to rate how much you were trying to get pregnant or avoid pregnancy right before you got pregnant, how would you rate yourself?" Respondents were then shown an eleven-point scale on a card and 
asked to point to a number from 0 to 10 , with a zero indicating they were trying hard not to get pregnant and a ten indicating they were trying hard to get pregnant. About one-quarter of the women selected scores of 1, 2, or 3, indicating they were contracepting with at least some regularity. About two-fifths of the women selected scores of 8,9 and 10, indicating they were procepting. Finally, about one-third of the women selected scores of 4 through 7 and within that onethird, about half selected a mid-point score of 5 . These women were probably not contracepting, at least not with any regularity, and were not procepting either. A similar picture is given by the results from the National Survey of Fertility Barriers conducted in the US between 2004 and 2006 (McQuillan et al. 2011), a telephone survey with an oversampling of women with a potential biomedical fertility barrier, but with the data weighted to make it nationally representative. Respondents were asked the following question: "Currently, are you pregnant, trying to get pregnant, trying not to get pregnant, or are you okay either way?" Of the 3,771 not currently pregnant women, $6 \%$ were trying to get pregnant, $71 \%$ were trying not to get pregnant and $23 \%$ were okay either way. The results from these two surveys suggest that there is a large group of women in the US who are neither contracepting regularly nor procepting, women who in all likelihood fall in the space in Figure 1 between $C_{1}$ and $0_{\mathrm{P}}$, where contraceptive and proceptive intent are weak or non-existent.

I have called the pregnancies that occur to this group of women "subintended" (Miller 2007), the idea being that these pregnancies are often affected by motivations that do not rise to the level of reflecting conscious decisions to pursue a particular target (having a baby, not having a baby) and thereby being identifiable as intentions. I would argue that this group of pregnancies - and there appear to be a great many of them, if the cited survey data are valid - are currently not well addressed by the conscious decision-making approach of the TPB. Bargh and Morsella (2008) have written a highly relevant paper called "The Unconscious Mind". These authors argue against the "conscious-centric" bias of many cognitive psychologists who see the unconscious mind as less flexible, deliberative and action-oriented than its conscious counterpart. They point to the evidence from contemporary social cognition research that demonstrates the existence of several unconscious behavioural guidance systems, including those that are perceptual, evaluative and motivational, and they endorse the notion that impulses to act are unconsciously activated and that the role of consciousness may largely be that of a gatekeeper and "after-the-fact sense maker". It would seem that this perspective may be a fruitful one with respect to the many pregnancies that are neither intended nor counterintended. Some psychologists who embrace the TPB in their research have made use of this perspective in their work (e.g. Gollwitzer and Bargh 2005), but I do not see that it has been made central to the TPB in a way that could help address a very important and common phenomenon, the subintended pregnancy. 


\section{Desires}

I have discussed this construct at length elsewhere in this volume (Miller 2011) and so will limit my comments here. Perhaps the most important point to be made is that this construct is present in the T-D-I-B framework but not in the TPB. The most closely analogous construct from the TPB is that of the behavioural attitude, which is simply how favourably or unfavourably a behaviour is evaluated. However there is a substantial difference between desires and behavioural attitudes with regard to how they function in the prediction of fertility. In the T-D-I-B framework desires are directed at having a child and specifically at the three actionable goals that are decisional determinants of fertility, namely childbearing, child-number and child-timing desires. In the TPB a distinction is made between giving birth (the action) and having a child (the target); behavioural attitudes are directed toward the action. This distinction seems somewhat awkward to me. For one thing, giving birth does not seem like a behaviour that implements having a child in the same way that contraceptive use implements not having one. For another thing, it does not seem that how a person evaluates giving birth is the primary driving motivational force in childbearing (although it is one such force) but rather how the person evaluates having a child. It should be noted that the same two difficulties arise with respect to intentions, which can also be formulated in terms of the three childbearing, child-number and child-timing goals.

One of the advantages of the desires construct is that, like motivations but unlike intentions, it can be formulated as two unipolar scales that have extensive overlap in the behavioural categories to which it applies. This can be seen in Figure 2, which shows both the hypothesised range for negative desires, which extends from approximately 50 to $100 \%$ strength for the highly effective contraceptive users to approximately 0 to $50 \%$ strength for the active proceptors, and the hypothesised range for positive desires, which extends from approximately 50 to $100 \%$ strength for the active proceptors to approximately 0 to $50 \%$ strength for the highly effective contraceptive users. The figure makes clear that there are large areas where the opposing desires overlap with equal or almost equal strength, suggesting an important role for ambivalent desires in the occurrence of unintended pregnancies. Although I am aware of some TPB researchers taking ambivalent motivations into consideration, I do not know of any work that systematically integrates interactions between positive and negative motivational variables within the mathematical structure of the theory the way it is beginning to be done within the T-D-I-B framework (Miller et al. 2011). 
Figure 2:

Ranges in the strength of both contraceptive desires $\left(D_{C}\right.$, dashed lines) and proceptive desires $\left(D_{P}\right.$, solid lines), based on hypothetical values assigned to each of eight categories of conception-oriented behaviour. The ranges for positive and negative intentions from Figure 1 are shown as faint dashed and solid lines

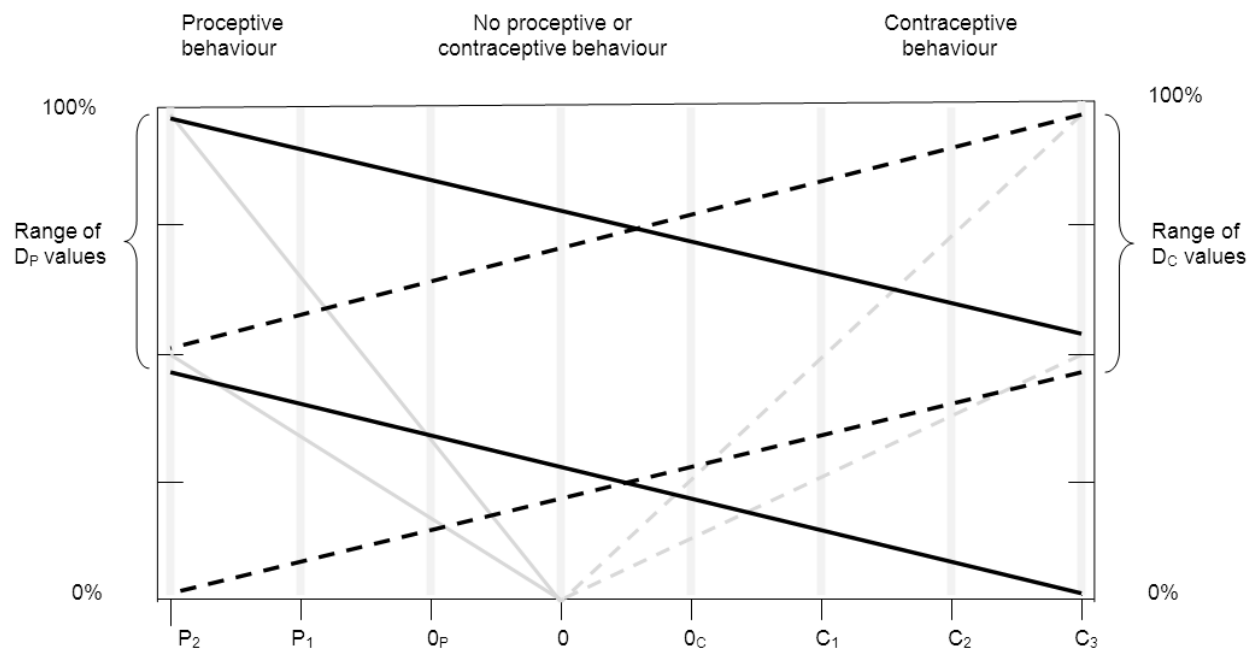

\section{Motivational traits}

In the T-D-I-B framework motivational traits are conceptualised in terms of bonding schemas. These are biologically based neural structures that organise the perceptions we have of other people in our social environment, how we feel and think about them, and the actions we take in our relationships with them. Such schemas are based on groups of neural networks that are preadapted to promote social bonding; that is to say, they have been constructed and shaped by evolution so that they function in ways that enable the human animal to survive and reproduce by living cooperatively in social groups (Miller and Rodgers 2001). The bonding schema of greatest relevance to childbearing is the nurturant schema (Miller 2003). The principal affective components of nurturant schemas are affection and fear, which respectively underlie the separate positive and negative motivations that drive the affection for and protection of dependent offspring. These affects, together with those that motivate the non-reproductive domains that compete with the bearing and rearing of children, constitute the principal motivational substrate for childbearing. I have developed a Childbearing Questionnaire (CBQ) that measures the positive and negative components as separate traits (Miller 1995) and have used the subscales of these two measures to successfully predict childbearing desires (Miller 1994) and child-number desires (Miller and Pasta 1993). 
It is useful to make a distinction between intrinsic motivations that have inherent rewards or punishments and extrinsic motivations that have instrumental rewards or punishments. With intrinsic motivations, the goal or target is inherently rewarding or punishing; with instrumental motivations, the goal or target is a means to some other source of reward or punishment. Most of the items of the CBQ can be viewed as intrinsic, for example "Holding and cuddling a baby" and "Guiding and teaching my child". However, one subscale in particular, appropriately named Instrumental Values of Children, only has extrinsic items, for example "Knowing that I am fertile" and "Having my family and friends admire me with my baby". Respondents taking the CBQ rate these items by indicating on a four-point scale how desirable each one is to them. It has always seemed to me that in the case of the CBQ's intrinsic items this rating procedure adequately covered both the evaluative and the subjective probability component of the behavioural belief construct that is central to the TPB approach, whereas for the extrinsic items the use of information about subjective probability might well add predictive strength to that of the desirability rating. Put somewhat differently, it has seemed to me that the TPB treated every value as an instrumental value and that not only did the approach in the CBQ offer a good alternative, especially for its intrinsic items, but it also avoided certain methodological costs, such as respondent fatigue and the uncertainty associated with variables based on multiplicative combinations (Ajzen and Fishbein 2008). Ultimately, of course, this is an empirical issue. I have collected probability data for the Childbearing Questionnaire and at some point it would be instructive to see whether using these would improve prediction for the intrinsic or extrinsic items.

\section{Other TPB components}

Although the TPB and the T-D-I-B framework both incorporate what others think and feel into their models of individual fertility behaviour and its outcomes, their respective approaches are quite different. The TPB has two possible routes of social influence, both acting on the person's fertility intentions. The primary route is via subjective social norms, which are based on the person's expectation that one or more social referents would approve of the behaviour in question, weighed by the person's motivation to comply with the referent(s). The other route involves perceived behavioural control in cases where a social referent is perceived as being someone who can impede or facilitate performance of the fertility behaviour. There is a weighing of beliefs about the referents being present by an evaluation of their power to impede or facilitate.

The T-D-I-B approach is different in a number of respects. First, it is based on the idea of nested systems within a general systems theory approach. Thus biological systems are viewed as underlying the psychology and behaviour of the individual organism and the individual is viewed as being part of multiple dyadic and more complex social systems (Miller 1979). Second, it assumes that as a 
result of affiliative and sexual bonding, the most important social influence on the individual actor's reproductive behaviour comes from that person's primary sexual partner or, in some cases, several partners. It follows from this assumption that reproductive behaviour typically involves joint decision-making and a cooperative pursuit, or avoidance, of pregnancy. It also follows that conflicts of interest are always present in sexually intimate dyads, threatening to produce individual decision-making and self-oriented behaviour. Third, it assumes that the best way to model these kinds of dyadic interactions is by measuring the motivational traits, desires and intentions of both members of the primary dyad (Miller et al. 2004) or, lacking the opportunity to obtain data from the other individual, by measuring the person's perception of the partner's motivational traits, desires and intentions. The latter alternative draws upon the individual's theory of mind and empathy capabilities and the underlying neural networks that support them (Shamay-Tsoory 2011). Obviously, the individual's other relationships, such as with family members or close friends, will affect decisionmaking and behaviour as well. However, given the primacy of the sexual partner in reproductive behaviour, it does not seem to me that motivation to comply with that partner adequately describes some of the effects of dyadic motivational conflict, which include the influence and disagreement effects of couple decisional conflict (Miller et al. 2004) and differences between the effects of positive and negative disagreement (Miller and Pasta 1996b).

Finally, it should be noted that self-efficacy is an important and successful part of the TPB that is not explicitly included in the T-D-I-B framework. Where a person's goal is to achieve conception, fecundity is probably the main factor impairing perceived behavioural control. Where the goal is to avoid conception, many factors are likely to be at work. It is of interest to speculate whether many of that large group who say they are okay either way with conceiving or not conceiving may perceive themselves as having impaired behavioural control about both of these outcomes.

\section{References}

Ajzen, I. 2010. Fertility intentions and the theory of planned behavior. Paper presented at the conference "From intentions to behavior: reproductive decision-making in a macro-micro perspective“. Vienna, Austria. 2-3 December 2010. http://www.oeaw.ac.at/vid/in2b/

Ajzen, I. and M. Fishbein 2008. Scaling and testing multiplicative combinations in the expectancy-value model of attitudes. Journal of Applied Social Psychology 38(9): 2222-2247.

Albarracin, D., B.T. Johnson, M. Fishbein and P.A. Muellerleile 2001. Theories of reasoned action and planned behavior as models of condom use: a meta-analysis. Psychological Bulletin 127(1): 142-161. 
Bachrach, C.A. and S. Newcomer 1999. Intended pregnancies and unintended pregnancies: distinct categories or opposite ends of a continuum? Family Planning Perspectives 31(5): 251-252.

Bargh, J.A. and E. Morsella 2008. The unconscious mind. Perspectives in Psychological Science 3(1): 73-79.

Finer, L.B., L.D. Lindberg, S. Singh and C. Stokes-Prindle 2007. Unpublished report to expert panel on pregnancy wantedness. Washington, DC, 10 May.

Gollwitzer, P.M. and J.A. Bargh 2005. Automaticity in goal pursuit. In Handbook of competence and motivation, ed. A. Elliot and C. Dwek, 624-646. New York: Guillford Press.

McQuillan, J., A.L. Greil and K.M. Shreffler 2011. Pregnancy intentions among women who do not try: focusing on women who are okay either way. Maternal and Child Health Journal 15(2): 178-187.

Miller, W.B. 1979. Psychosocial aspects of contraception. In Contraception: science, technology, and application, ed. National Research Council, 269-287. Washington, DC: National Academy of Sciences.

Miller, W.B. 1986. Proception: an important fertility behavior. Demography 23(1): 579-594.

Miller, W.B. 1994. Childbearing motivations, desires, and intentions: a theoretical framework. Genetic, Social, and General Psychological Monographs 120(2): 223-258.

Miller, W.B. 1995. Childbearing motivation and its measurement. Journal of Biosocial Science 27: 473-485.

Miller, W.B. 2003. The role of nurturant schemas in human reproduction. In The biodemography of fertility, ed. J.L. Rodgers and H.-P. Kohler, 43-55. Boston: Kluwer.

Miller, W.B. 2007. Fertility intentions, counterintentions, and subintentions: a theoretical framework and graphic model www.tfri.org//_TFRI.org_/TFRI_Unpublished_Work.html

Miller, W.B. 2011. Differences between fertility desires and intentions: implications for theory, research, and policy. Vienna Yearbook of Population Research Vol. 9:75-98.

Miller, W.B., J.S. Barber and H.H. Gatney 2011. Ambivalent fertility desires: effects on pregnancy risk in young women. Under review.

Miller, W.B. and D.J. Pasta 1993. Motivational and nonmotivational determinants of child-number desires. Population and Environment 15(2): 113-138.

Miller, W.B. and D.J. Pasta 1995. Behavioral intentions: which ones predict fertility behavior in married couples? Journal of Applied Social Psychology 25(6): 530-555.

Miller, W.B. and D.J. Pasta 1996a. Patterns of proceptive behavior in a non-clinical sample of married couples. www.tfri.org//_TFRI.org_TFRI_Unpublished_Work.html

Miller, W.B. and D.J. Pasta 1996b. Couple disagreement: effects on the formation and implementation of fertility decisions. Personal Relationships 3: 307-336.

Miller, W.B. and D.J. Pasta 1996c. The relative influence of husbands and wives on the choice and use of oral contraception, a diaphragm, and condoms. Journal of Applied Social Psychology 26: 1749-1774.

Miller, W.B., D.J. and Pasta 2002. The motivational substrate of unintended and unwanted pregnancy. Journal of Applied Biobehavioral Research 7(1): 1-29.

Miller, W.B. and J.L. Rodgers 2001. The ontogeny of human bonding systems. Boston: Kluwer. 
Miller, W.B., L.J. Severy and D.J. Pasta 2004. A framework for modeling fertility motivation in the dyad. Population Studies 58(2): 193-205.

Miller, W.B., M. Trent, and S. Chung 2011. Ambivalent childbearing motivation and condom use in young urban women. Presented at the Psychosocial Workshop, Washington, DC, 29-30 March 2011. www.tfri.org//_TFRI.org_/TFRI_Unpublished_Work.html

Philipov, D., O. Thévenon, J. Klobas, L. Bernardi, and A.C. Liefbroer 2009. Reproductive decision-making in a macro-micro perspective (REPRO): a state of the art review. http://www.oeaw.ac.at/vid/download/edrp_1_09.pdf

Shamay-Tsoory, S.G. 2011. Empathic processing: its cognitive and affective dimensions and neuroanatomical basis. In The social neuroscience of empathy, ed. J. Decety and W. Ickes, 215-232. Cambridge, MA: Bradford Books.

Sutton, S., D. McVey and A. Glanz 1999. A comparative test of the theory of reasoned action and the theory of planned behavior in the prediction of condom use intentions in a national sample of English young people. Health Psychology 18(10): 72-81.

Vienna Institute of Demography 2010. From intentions to behavior: reproductive decision-making in a macro-micro perspective. http://www.oeaw.ac.at/vid/in2b/ 
